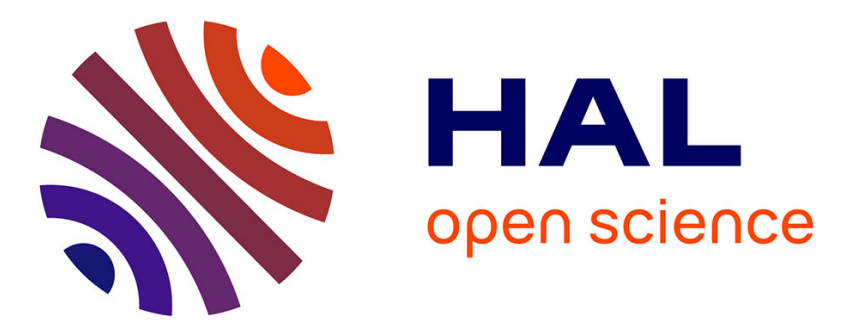

\title{
Differential Flatness and Control of Protocentric Aerial Manipulators with Any Number of Arms and Mixed Rigid-/Elastic-Joints
}

\author{
Burak Yüksel, Gabriele Buondonno, Antonio Franchi
}

\section{- To cite this version:}

Burak Yüksel, Gabriele Buondonno, Antonio Franchi. Differential Flatness and Control of Protocentric Aerial Manipulators with Any Number of Arms and Mixed Rigid-/Elastic-Joints. IEEE/RSJ International Conference on Intelligent Robots and Systems, Oct 2016, Daejeon, South Korea. hal01350867

\section{HAL Id: hal-01350867 \\ https://hal.science/hal-01350867}

Submitted on 2 Aug 2016

HAL is a multi-disciplinary open access archive for the deposit and dissemination of scientific research documents, whether they are published or not. The documents may come from teaching and research institutions in France or abroad, or from public or private research centers.
L'archive ouverte pluridisciplinaire HAL, est destinée au dépôt et à la diffusion de documents scientifiques de niveau recherche, publiés ou non, émanant des établissements d'enseignement et de recherche français ou étrangers, des laboratoires publics ou privés. 


\title{
Differential Flatness and Control of Protocentric Aerial Manipulators with Any Number of Arms and Mixed Rigid-/Elastic-Joints
}

\author{
Burak Yüksel $^{1}$, Gabriele Buondonno ${ }^{2}$ and Antonio Franchi ${ }^{3}$
}

\begin{abstract}
In this paper we introduce a particularly relevant class of aerial manipulators that we name protocentric. These robots are formed by an underactuated aerial vehicle, a planarVertical Take-Off and Landing (PVTOL), equipped with any number of different parallel manipulator arms with the only property that all the first joints are attached at the Center of Mass (CoM) of the PVTOL, while the center of actuation of the PVTOL can be anywhere. We prove that protocentric aerial manipulators (PAMs) are differentially flat systems regardless the number of joints of each arm and their kinematic and dynamic parameters. The set of flat outputs is constituted by the CoM of the PVTOL and the absolute orientation angles of all the links. The relative degree of each output is equal to four. More amazingly, we prove that PAMs are differentially flat even in the case that any number of the joints are elastic, no matter the internal distribution between elastic and rigid joints. The set of flat outputs is the same but in this case the total relative degree grows quadratically with the number of elastic joints. We validate the theory by simulating object grasping and transportation tasks with unknown mass and parameters and using a controller based on dynamic feedback linearization.
\end{abstract}

\section{INTRODUCTION}

Systems consisting of aerial vehicles and manipulator arms have been increasingly studied within the last years, as they enjoy the great workspace of the flying robot base, and the dexterity of the manipulator arm attached to it. The major implementation of such systems are aerial physical interaction, transportation and manipulation [1], [2].

Control of the aerial robots with a fixed rigid link as flying tools is presented in [3], and this work is improved to the multiple flying aerial vehicles case in [4]. In [5], the authors showed an adaptive sliding mode technique for controlling a quadrotor UAV equipped with a twodegrees-of-freedom (2-DoF) rigid arm. Dynamic modeling of a quadrotor with a redundant and fully rigid arm using passive decomposition technique is studied in [6]. In a greater scale, the authors of [7] showed the experimental results of controlling an industrial size manipulator attached on an autonomous helicopter. In a smaller scale, a behavioral control framework for aerial vehicles together with a lightweight manipulator arm is presented in [8]. In [9] a quadrotor equipped with two manipulator arms is presented, which is used to perform a valve opening task. In all these works, one

\footnotetext{
${ }^{1}$ Max Planck Institute for Biological Cybernetics, Spemanstr. 38, 72076, Tübingen, Germany. burak • yueksel@tuebingen.mpg. de

${ }^{2}$ Dipartimento di Ingegneria Informatica, Automatica e Gestionale, Sapienza Università di Roma, Via Ariosto 25, 00185 Roma, Italy. buondonno@diag. uniroma1. it

${ }^{3}$ LAAS-CNRS, Université de Toulouse, CNRS, Toulouse, France, afranchi@laas.fr

This work has been partially funded by the European Union's Horizon 2020 research and innovation programme under grant agreement No 644271 AEROARMS
}

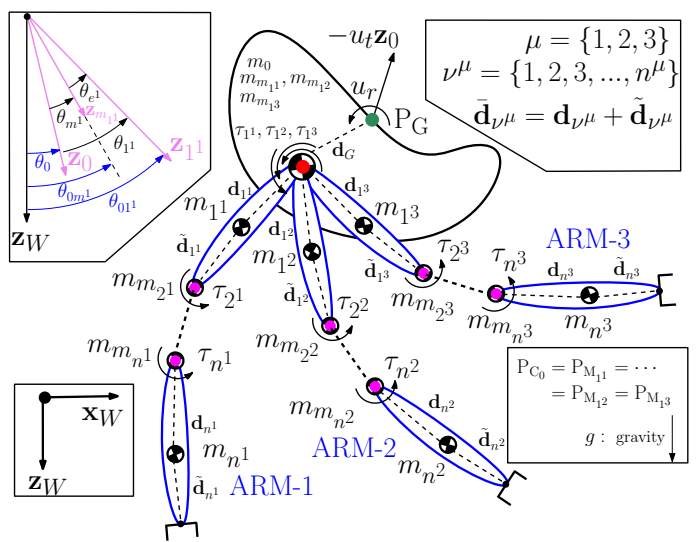

Fig. 1: Sketch of a protocentric aerial manipulator (PAM) for $m=3$. On the left up relative and absolute angles of the motor and the link of the first joint of the first manipulator are depicted, where the length of the $z$ axes are made different just for illustration purposes.

or multiple manipulator arms with rigid actuators are used on underactuated aerial platforms. To our best knowledge, a compliant arm on an underacuated aerial vehicle was first time considered in [10], and followed very recently by [11].

Although aerial robots with manipulators have recently become an interest of the researchers, the research on the manipulator arms itself (on a fixed-base) has been deeply studied and intensively developed over the last several decades [12]. We especially have good knowledge on the system characteristics of the fixed-base manipulators, e.g., differential flatness properties. This property allows to know in advance (algebraically) the nominal state and the input trajectories along which the system will evolve while tracking a desired output trajectory [13], which is very useful especially in the planning phase. Moreover it is well known that differential flatness implies input-to-state linearizability via dynamic feedback in an open and dense set of the state space and that a flat output is exactly linearizing [14]. Exact linearizability of grounded manipulator arms have been studied, when the joint connection is rigid [15], elastic [16] or both of them [17]. More interesting studies on controlling arms with compliance can be found in [18].

The differential flatness and control of quadrotor UAVs have been studied previously by different groups, e.g. [19]. In [20], the authors studied the case of a planar-Vertical Take-Off and Landing (PVTOL) vehicle equipped with a one DoF rigid arm. This result has been recently extended generalized in a few directions by [11].

As for the flying vehicle we consider the case of a (vertical) planar-VTOL (PVTOL) aerial platform, similar to previous studies (see, e.g., [21], [22]) in the aerial robotics 
field. This reduced system does not only capture the nonlinear features and the underactuation of a 3D system, but also allows to generalize the obtained results in a later stage. Furthermore, many practical aerial problems are, fundamentally, $2 \mathrm{D}$ problems immersed in a $3 \mathrm{D}$ world.

In this paper, we present a generic dynamic model of a PVTOL equipped with multiple arms, each having a possibly different number of links with any distribution of rigid or elastic joints. We consider the case of protocentric aerial manipulators (PAM), where all the arms are attached to the CoM of the PVTOL. We show that PAMs are differentially flat systems both when all the joints of the arms are rigid and also when any number of them are elastic. A surprising fact fact is that, contrarily to the case of a robotic arm attached to a fixed base [16], the total relative degree grows quadratically with the number of elastic joints, due to the underactuation of the flying base. This fact makes the control of aerial manipulators with elastic joints a very challenging task. Finally, we present an exact linearizing controller for tracking in the case that all the joints are rigid.

The detailed derivations of the model and proofs are presented as a technical report in [23] due to the page limit.

\section{Definitions And Assumptions}

In order to model a generic Protocentric Aerial Manipulator (PAM), we start with the following definitions:

- A PAM is constituted by a PVTOL with attached $m \geq 1$ manipulating arms (see Fig. 1 where $m=3$ ); an arbitrary arm is called the $\mu$-th arm, where $\mu \in\{1,2,3, \cdots, m\}$.

- The $\mu$-th arm is constituted by $n^{\mu}$ joint/motor/link elements; an arbitrary joint, motor, or link is called the $v^{\mu}$-th joint, motor, or link, where $v^{\mu} \in\left\{1,2,3, \cdots, n^{\mu}\right\}$. We also define the total number of joints $n=\sum_{i=\mu}^{m} n^{\mu}$.

- The $\mu$-th arm has $k^{\mu}$ elastic joints; an arbitrary elastic joint is called the $\kappa^{\mu}$-th elastic joint, where $\kappa^{\mu} \in$ $\left\{1,2,3, \cdots, k^{\mu}\right\}$. Similarly, we define $k=\sum_{i=\mu}^{m} k^{\mu}$.

- It is always $0 \leq k^{\mu} \leq n^{\mu}$ and $0 \leq k \leq n$.

With this convention, we call, e.g., the mass of the $v$-th link of the $\mu$-th arm as $m_{v^{\mu}}$, or the motor rotational inertia of the $v$-th link of the $\mu$-th arm is called as $J_{m_{v} \mu}$ (i.e., the subscript corresponds to the joint, and the superscript to the arm).

The following assumptions are then made:

A1. Only the 2D dynamics of a PVTOL aerial vehicle with $m$ different fully actuated robotic arms is considered .

A2. All the joints are actuated via a motor, and the rotational center of this motor is the same with the center of the revolute joint that is attached to it.

A3. [Protocentricity] The first joint of each robotic arm is placed at the Center of Mass (CoM) of the PVTOL, i.e., $\mathrm{P}_{\mathrm{C}_{0}}=\mathrm{P}_{\mathrm{M}_{1^{1}}}=\mathrm{P}_{\mathrm{M}_{1^{2}}}=\cdots=\mathrm{P}_{\mathrm{M}_{1 \mathrm{~m}}}$ (see also Fig. 1 ).

A4. Each motor is attached to the next link in the chain either rigidly or via some elastic joint.

We denote with $\mathscr{F}_{W}:\left\{\mathrm{P}_{\mathrm{W}}, \mathbf{x}_{W}, \mathbf{z}_{W}\right\}$ and $\mathscr{F}_{0}:\left\{\mathrm{P}_{\mathrm{C}_{0}}, \mathbf{x}_{0}, \mathbf{z}_{0}\right\}$ the world (inertial) frame and the frame attached to the PVTOL, respectively, where $\mathrm{P}_{\mathrm{C}_{0}}$ is the Center of Mass (CoM) of the PVTOL. Define $\mathrm{P}_{\mathrm{M}_{\mathrm{v}} \mu}$ as the center of the $v^{\mu}$-th motor. The $v^{\mu}$-th joint and motor rotate about an axis parallel to $\mathbf{z}_{W} \times \mathbf{x}_{W}$ and passing through $\mathrm{P}_{\mathrm{M}_{\mathrm{v}} \mu}$. The $v^{\mu}$-th motor frame is denoted with $\mathscr{F}_{M_{v} \mu}:\left\{\mathrm{P}_{\mathrm{M}_{v} \mu}, \mathbf{x}_{m_{v} \mu}, \mathbf{z}_{m_{v} \mu}\right\}$ and it is rigidly attached to the output shaft of the $v^{\mu}$-th motor. We consider also the $v^{\mu}$-th link frame $\mathscr{F}_{v^{\mu}}:\left\{\mathrm{P}_{\mathrm{C}^{\mu}}, \mathbf{x}_{v^{\mu}}, \mathbf{z}_{v^{\mu}}\right\}$, where $\mathrm{P}_{\mathrm{C}_{\nu \mu} \mu}$ is the CoM of the $v^{\mu}$-th link. Finally we denote with $\mathrm{P}_{\mathrm{E}^{\mu}}$ the terminal point of the end-effector of the $\mu$-th arm, and with $\mathrm{P}_{\mathrm{C}}$ the $\mathrm{CoM}$ of the whole robotic system (i.e., the PVTOL plus all the arms).

Given an angle $\theta_{*} \in \mathbb{R}$ between the z-axes of two frames (see Fig. 1, top left side) the usual rotation matrix definition $\mathbf{R}_{*} \in \mathrm{SO}(2)$ holds. Therefore, the orientations of, e.g., $\mathscr{F}_{0}$ in $\mathscr{F}_{W}$, and $\mathscr{F}_{v^{\mu}}$ in $\mathscr{F}_{0}$ are expressed by the rotation matrices $\mathbf{R}_{0}$, and $\mathbf{R}_{v^{\mu}}$, respectively. The absolute angles of the $v^{\mu}$ th motor and link are $\theta_{0 m_{v} \mu}=\theta_{0}+\sum_{i^{\mu}=1}^{v^{\mu}} \theta_{m_{i} \mu}$ and $\theta_{0 v^{\mu}}=$ $\theta_{0}+\sum_{i^{\mu}=1}^{v^{\mu}} \theta_{i \mu}$, respectively (see Fig. 1, top left side). Notice that $\theta_{e_{v} \mu}=\theta_{v^{\mu}}-\theta_{m_{v} \mu}=\theta_{0 v^{\mu}}-\theta_{0 m_{v} \mu}$ is constantly zero if the $v^{\mu}$ joint is rigid and can be any if it is elastic.

The constant position of $\mathrm{P}_{\mathrm{M}_{v} \mu}$ and of $\mathrm{P}_{\mathrm{M}_{(v+1)} \mu}$ in $\mathscr{F}_{v^{\mu}}$ are denoted with $-\mathbf{d}_{v^{\mu}}=\left[-d_{v^{\mu}}-d_{v^{\mu}}\right]^{T} \in \mathbb{R}^{2}$ and

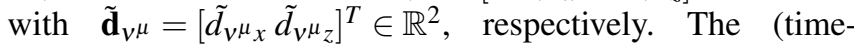
varying) positions of $\mathrm{P}_{\mathrm{C}}, \mathrm{P}_{\mathrm{C}_{0}}, \mathrm{P}_{\mathrm{C}_{\nu} \mu}, \mathrm{P}_{\mathrm{M}_{\nu} \mu}$ and $\mathrm{P}_{\mathrm{E}^{\mu}}$ in $\mathscr{F}_{W}$ are denoted with $\mathbf{p}_{c}=\left[x_{c} z_{c}\right]^{T} \in \mathbb{R}^{2}, \mathbf{p}_{0}=\left[x_{0} z_{0}\right]^{T} \in \mathbb{R}^{2}$, $\mathbf{p}_{v^{\mu}}=\left[x_{v^{\mu}} z_{v^{\mu}}\right]^{T} \in \mathbb{R}^{2}, \mathbf{p}_{m_{\gamma^{\mu}}}=\left[x_{m_{v^{\mu}}} z_{m_{\gamma^{\mu}}}\right]^{T} \in \mathbb{R}^{2}$, and $\mathbf{p}_{e^{\mu}}=$

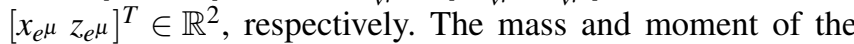
inertia of the PVTOL and the $v^{\mu}$-th motor and link are denoted with $m_{0} \in \mathbb{R}_{>0}, J_{0} \in \mathbb{R}_{>0} ; m_{m_{\gamma^{\mu}}} \in \mathbb{R}_{>0}, J_{m_{y} \mu} \in$ $\mathbb{R}_{>0} ; m_{v^{\mu}} \in \mathbb{R}_{>0}, J_{v^{\mu}} \in \mathbb{R}_{>0}$, respectively. The gravitational constant is $g \in \mathbb{R}^{+}$. Also $m_{s}=m_{0}+\sum_{\mu=1}^{m} \sum_{v=1}^{n^{\mu}}\left(m_{m_{v \mu}}+m_{v^{\mu}}\right)$ is the total mass of the overall system.

The point $\mathrm{P}_{\mathrm{G}}$ is the center of actuation of the PVTOL (green point in Fig 1). The constant position of $\mathrm{P}_{\mathrm{G}}$ in $\mathscr{F}_{0}$ is denoted with $\mathbf{d}_{G}=\left[d_{G_{x}} d_{G_{z}}\right]^{T} \in \mathbb{R}^{2}$. The PVTOL is actuated by means of: i) a total thrust force $-u_{t} \mathbf{z}_{0} \in \mathbb{R}^{2}$ applied at $P_{G}$, where $u_{t} \in \mathbb{R}$ is its magnitude, and ii) a total torque (moment) $u_{r}\left(\mathbf{z}_{0} \times \mathbf{x}_{0}\right) \in \mathbb{R}^{2}$ applied also at $P_{G}$, where $u_{r} \in \mathbb{R}$ is the torque intensity. Furthermore, an individual motor for each joint applies a torque $\tau_{v^{\mu}}\left(\mathbf{z}_{v^{\mu}} \times \mathbf{x}_{v^{\mu}}\right)$ at $\mathrm{P}_{\mathrm{M}_{v^{\mu}}}$ to the joint, where $\tau_{\nu^{\mu}} \in \mathbb{R}$ is its intensity.

\section{CASE R: Dynamics With Rigid JoInts ONLY}

Let us first consider the case in which all the joints are rigid, i.e., $k=0$. The aerial manipulator has therefore $3+n$ degrees of Freedom (DoFs) corresponding to the generalized coordinates $\mathbf{q}=\left[\mathbf{q}_{p}^{T} \mathbf{q}_{r}^{T}\right]^{T} \in \mathbb{R}^{(3+n)}$ where $\mathbf{q}_{p}$ are the PVTOL coordinates, and $\mathbf{q}_{r}$ are the arm-side coordinates:

$$
\begin{aligned}
\mathbf{q}_{p} & =\left[\begin{array}{ll}
\mathbf{p}_{0}^{T} & \theta_{0}
\end{array}\right]^{T} \in \mathbb{R}^{3} \\
\mathbf{q}_{r} & =\left[\begin{array}{lll}
\mathbf{q}_{r^{1}}^{T} & \cdots & \mathbf{q}_{r^{m}}^{T}
\end{array}\right]^{T} \in \mathbb{R}^{n}, \mathbf{q}_{r^{\mu}}^{T}=\left[\begin{array}{lll}
\theta_{01^{\mu}} & \cdots & \theta_{0 n^{\mu}}
\end{array}\right]^{T} \in \mathbb{R}^{n^{\mu}} .
\end{aligned}
$$

Then using the Lagrange equation and after some straightforward algebra (see Sec. II-A of [23] for details), we can find the generalized inertia matrix as

$$
\begin{gathered}
\mathbf{M}=\left(\begin{array}{cc}
\mathbf{M}_{p} & * \\
\mathbf{M}_{p r} & \mathbf{M}_{r}
\end{array}\right)=\mathbf{M}^{T} \in \mathbb{R}^{(3+n) \times(3+n)} \\
\mathbf{M}_{p}=\operatorname{diag}\left(m_{s} m_{s} J_{0}\right), \quad \mathbf{M}_{p r}=\left(\begin{array}{lll}
\mathbf{M}_{p r^{1}}^{T} & \ldots & \mathbf{M}_{p r^{m}}^{T}
\end{array}\right)^{T} \\
\mathbf{M}_{p r^{\mu}}=\left(\begin{array}{cc}
\mathbf{m}_{01} \mu\left(\theta_{01} \mu\right)^{T} & 0 \\
\vdots & \vdots \\
\mathbf{m}_{0 n^{\mu}}\left(\dot{\theta}_{0 n^{\mu}}\right)^{T} & 0
\end{array}\right) \in \mathbb{R}^{n^{\mu} \times 3},
\end{gathered}
$$


where $m_{s}$ is the total mass, $\mathbf{M}_{p} \in \mathbb{R}^{3 \times 3}$ is the PVTOL side inertia matrix, $\mathbf{M}_{r}\left(\mathbf{q}_{r}\right) \in \mathbb{R}^{n \times n}$ is the manipulator side inertia matrix, and $\mathbf{M}_{p r}\left(\mathbf{q}_{r}\right) \in \mathbb{R}^{n \times 3}$ represents the inertial couplings between the PVTOL and the manipulator arms. More details on the computation are given in Sec.II-B of [23].

The gravitational forces are the following

$$
\mathbf{g}=\left[\begin{array}{ll}
\mathbf{g}_{p}^{T} & \mathbf{g}_{r}^{T}
\end{array}\right]^{T} \in \mathbb{R}^{(3+n)}, \mathbf{g}_{p}=\left[\begin{array}{lll}
0 & -m_{s} g & 0
\end{array}\right]^{T} \in \mathbb{R}^{3}
$$

where $\mathbf{g}_{r}=\left[\mathbf{g}_{r^{1}}^{T} \cdots \mathbf{g}_{r^{m}}^{T}\right]^{T} \in \mathbb{R}^{n}$ and for the $\mu$-th manipulator;

$$
\mathbf{g}_{r^{\mu}}=\left[\begin{array}{c}
-g \mathbf{m}_{01} \mu\left(\theta_{01} \mu\right)^{T} \mathbf{e}_{2} \\
\vdots \\
-g \mathbf{m}_{0 n} \mu\left(\theta_{0 n} \mu\right)^{T} \mathbf{e}_{2}
\end{array}\right] \in \mathbb{R}^{n^{\mu}} .
$$

with $\mathbf{e}_{2}=\left[\begin{array}{ll}0 & 1\end{array}\right]^{T}$. The Coriolis/centrifugal forces are found as

$$
\mathbf{c}=\left[\begin{array}{c}
\sum_{j=1}^{m} \sum_{i=1}^{n^{j}} \overline{\mathbf{m}}_{0 i j} \dot{\theta}_{0 i j}^{2} \\
0 \\
\mathbf{c}_{r}\left(\mathbf{q}_{r}, \dot{\mathbf{q}}_{r}\right)
\end{array}\right] \in \mathbb{R}^{(3+n) \times 1}
$$

where $\overline{\mathbf{m}}_{0 i \mu}=\frac{\partial \mathbf{m}_{0 i} \mu}{\partial \theta_{0 j} \mu} \in \mathbb{R}^{2 \times 1}$ and $\mathbf{c}_{r}\left(\mathbf{q}_{r}, \dot{\mathbf{q}}_{r}\right) \in \mathbb{R}^{n}$ are the arm side Coriolis forces. All the explicit steps for computing $\mathbf{g}$ and $\mathbf{c}$ can be found in Sec. II-C of [23].

Finally, the generalized forces are

$$
\begin{aligned}
\mathbf{f} & =\left[\begin{array}{c}
-u_{t} \sin \left(\theta_{0}\right) \\
-u_{t} \cos \left(\theta_{0}\right) \\
d_{G_{x}} u_{t}+u_{r}-\sum_{j=1}^{m} \tau_{1 j} \\
\overline{\mathbf{T}}
\end{array}\right]=\mathbf{G u} \in \mathbb{R}^{(n+3)}, \\
\overline{\mathbf{T}} & =\left[\begin{array}{llll}
\overline{\boldsymbol{\tau}}^{T} & \cdots & \overline{\boldsymbol{\tau}}^{m^{T}}
\end{array}\right]^{T} \in \mathbb{R}^{n} \\
\bar{\tau}^{\mu} & =\left[\begin{array}{llll}
\tau_{1} \mu-\tau_{2^{\mu}} & \cdots & \tau_{n^{\mu}-1}-\tau_{n^{\mu}} & \tau_{n^{\mu}}
\end{array}\right]^{T} \in \mathbb{R}^{n^{\mu}},
\end{aligned}
$$

which leads to a control input matrix of the following form

$$
\mathbf{G}=\left(\begin{array}{c|c|c}
\mathbf{v}\left(\theta_{0}\right) & \mathbf{0} & \mathbf{0} \\
\hline d_{G_{x}} & 1 & \mathbf{G}_{r p} \\
\hline \mathbf{0} & \mathbf{0} & \mathbf{G}_{r r}
\end{array}\right) \in \mathbb{R}^{(n+3) \times(n+2)},
$$

where $\mathbf{v}=-\mathbf{z}_{0} \in \mathbb{R}^{2}$, and all the other parts are explicitly given in Sec. II-D of [23]. The control input vector is

$$
\mathbf{u}=\left[\begin{array}{lllll}
u_{t} & u_{r} & \boldsymbol{\tau}^{1^{T}} & \boldsymbol{\tau}^{2^{T}} \cdots & \boldsymbol{\tau}^{m^{T}}
\end{array}\right]^{T} \in \mathbb{R}^{(n+2)},
$$

where $\tau^{\mu}=\left[\begin{array}{llll}\tau_{1^{\mu}} & \tau_{2^{\mu}} & \cdots & \tau_{n^{\mu}}\end{array}\right] \in \mathbb{R}^{n^{\mu}}$. Then finally the system dynamics can be written in the following form

$$
\mathbf{M} \ddot{\mathbf{q}}+\mathbf{c}+\mathbf{g}=\mathbf{G u} .
$$

The following result holds:

Proposition 1. $\mathbf{y}=\left[\mathbf{p}_{0}^{T} \mathbf{q}_{r}^{T}\right]^{T} \in \mathbb{R}^{(n+2)}$ is a flat output for the protocentric aerial manipulator with all rigid joints $(k=$ $0)$. The relative degree of each entry of $\mathbf{y}$ is 4 , and the total relative degree is $4 n+8$.

Proof. (Sketch) See Sec. I-A of [23] for the full proof. From the dynamics of the CoM of overall system one can show that $u_{t}$ and $\theta_{0}$ can be computed as functions of $\mathbf{y}, \dot{\mathbf{y}}$ and $\ddot{\mathbf{y}}$. (See (1)-(2) of [23]). The torque of the $v^{\mu}$-th motor is

$$
\begin{array}{r}
\tau_{v^{\mu}}=\tau_{v^{\mu}+1}+\mathbf{m}_{0 v^{\mu}}^{T}\left(\theta_{0 v^{\mu}}\right) \ddot{\mathbf{p}}_{0}+c_{r_{v^{\mu}}}\left(\mathbf{q}_{r^{\mu}}, \dot{\mathbf{q}}_{r^{\mu}}\right)+\mathbb{J}_{v^{\mu}} \ddot{\theta}_{0 v^{\mu}}+ \\
+g_{r_{v^{\mu}}}\left(\theta_{0 v^{\mu}}\right)+\sum_{l=1, l \neq v^{\mu}}^{n^{\mu}} m_{l v^{\mu}}\left(\theta_{0 l^{\mu}}, \theta_{0 v^{\mu}}\right) \ddot{\theta}_{0 l^{\mu}}, \quad \text { (8) }
\end{array}
$$

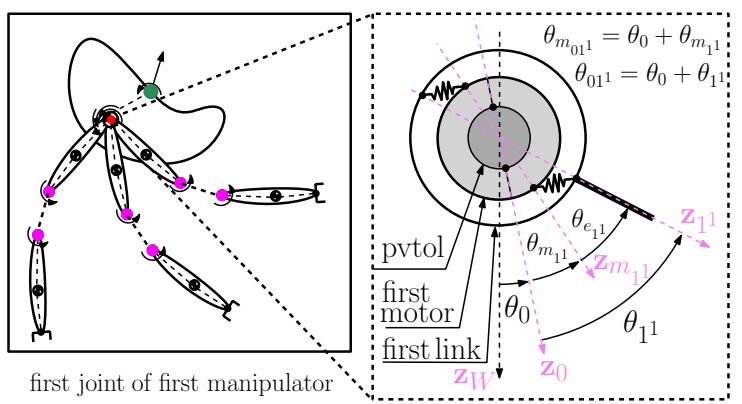

Fig. 2: An ideal example of elastic joint between the first motor shaft and the first link of the first arm on a PVTOL $(v=\mu=$ 1). Actuator is magnified w.r.t. the PVTOL. The innermost circle, fixed to $\mathscr{F}_{0}$, represents the PVTOL. The middle circle, fixed to $\mathscr{F}_{M_{11}}$, represents the actuator (or motor). The outermost circle is connected to the middle circle via elastic components, and it is rigidly connected to the link (fixed to $\mathscr{F}_{1^{1}}$.)

where $c_{r_{v} \mu}$ and $g_{r_{v} \mu}$ are the $v^{\mu}$-th elements of vectors $\mathbf{c}_{r}$ and $\mathbf{g}_{r}$ corresponding to the Coriolis and gravitational forces acting on the center of the $v^{\mu}$-th link, respectively (for $v^{\mu}=$ $n^{\mu}$ it is $\tau_{v^{\mu}+1}=0$ ). This means we can write the control torque of the $v^{\mu}$-th joint in the form of $\tau_{v^{\mu}}=\tau_{v^{\mu}}(\mathbf{y}, \dot{\mathbf{y}}, \ddot{\mathbf{y}})$. Finally the PVTOL torque is computed as

$$
u_{r}=\mathbb{J}_{0} \ddot{\theta}_{0}+\sum_{j=1}^{m} \tau_{1^{j}}-d_{G_{x}} u_{t},
$$

which means one can compute all the inputs and the states of the system as the functions of flat outputs and a finite number of its derivatives.

Also, since $\theta_{0}$ is a function of $\ddot{\mathbf{y}}$, then $\ddot{\theta}_{0}$ is a function of $\dddot{\mathbf{y}}$, and so is $u_{r}$, implying the relative degree of the system is four times the dimension of $\mathbf{y}$, i.e. $r=4(2+n)=8+4 n$.

\section{CASE E: Dynamics With Rigid/Elastic Joints}

We consider now the case $k \geq 1$, i.e., when at least one joint is elastic (see Fig. 2). The coordinates for the $\mu$-th manipulator are $\mathbf{q}_{r^{\mu}}=\left[\mathbf{q}_{r_{r}^{\mu}}^{T_{\mu}} \mathbf{q}_{r_{e}^{\mu}}^{T}\right]^{T} \in \mathbb{R}^{n^{\mu}+k^{\mu}}$, where $\mathbf{q}_{r_{r}} \in \mathbb{R}^{n^{\mu}}$ contains the orientations of the links and $\mathbf{q}_{r_{e}^{\mu}} \in \mathbb{R}^{k^{\mu}}$ contains the orientations of the motors that are connected to their links via an elastic joint. The full generalized coordinates

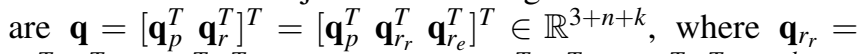
$\left[\mathbf{q}_{r_{r}^{1}}^{T} \mathbf{q}_{r_{r}^{2}}^{T} \cdots \mathbf{q}_{r_{r}^{m}}^{T}\right]^{T} \in \mathbb{R}^{n}$ and $\mathbf{q}_{r_{e}}=\left[\mathbf{q}_{r_{e}^{1}}^{T} \mathbf{q}_{r_{e}^{2}}^{T} \cdots \mathbf{q}_{r_{e}^{m}}^{T}\right]^{T} \in \mathbb{R}^{k}$.

Define the set of sets $N:=\left\{N^{1}, N^{2}, \cdots, N^{m}\right\}$, where $N^{\mu}:=$ $\left\{1,2, \cdots, n^{\mu}\right\}$, and the set of sets $K:=\left\{K^{1}, K^{2}, \cdots, K^{m}\right\}$, where $K^{\mu} \subset N^{\mu}$ is the sorted set of indexes of the elastic joints of the $\mu$-th arm. We denote with $\left\{\kappa^{\mu}\right\}$ the $\kappa^{\mu}$-th element of $K^{\mu}$, where $\kappa^{\mu}=1, \ldots,\left|K^{\mu}\right|=k^{\mu}$. Therefore, it is $\mathbf{q}_{r_{e}^{\mu}}=\left[\begin{array}{llll}\theta_{m_{0\left\{\kappa^{\mu}=1\right\}}} & \theta_{m_{0\left\{\kappa^{\mu}=2\right\}}} \cdots \theta_{\left.m_{0\left\{\kappa^{\mu}=k^{\mu}\right\}}\right\}}\end{array}\right]^{T} \in \mathbb{R}^{k^{\mu}}$.

For each arm $\mu$ we define i) the diagonal matrix $\mathbf{S}_{N^{\mu}} \in \mathbb{R}^{n^{\mu} \times n^{\mu}}$ whose $v^{\mu}$-th diagonal element is equal to 1 if $v^{\mu} \in K^{\mu}$ and zero otherwise, and ii) the selection matrix $\mathbf{S}_{K^{\mu}} \in \mathbb{R}^{k^{\mu} \times n^{\mu}}$ obtained from $\mathbf{S}_{N^{\mu}}$ by removing all the zero row vectors. Then we define the block diagonal matrices

$$
\begin{aligned}
& \mathbf{S}_{N}=\operatorname{diag}\left\{\mathbf{S}_{N^{1}}, \mathbf{S}_{N^{2}}, \cdots, \mathbf{S}_{N^{m}}\right\} \in \mathbb{R}^{n \times n}, \\
& \mathbf{S}_{K}=\operatorname{diag}\left\{\mathbf{S}_{K^{1}}, \mathbf{S}_{K^{2}}, \cdots, \mathbf{S}_{K^{m}}\right\} \in \mathbb{R}^{k \times n} .
\end{aligned}
$$

See Sec. II-E of [23] for examples of this notation.

Let us then first rewrite the generalized inertia matrix as 


$$
\mathbf{M}_{E}=\left(\begin{array}{ccc}
\mathbf{M}_{p} & * & * \\
\mathbf{M}_{p r} & \mathbf{M}_{r E} & * \\
\mathbf{0} & \mathbf{0} & \mathbf{D}_{K}
\end{array}\right)=\mathbf{M}^{T} \in \mathbb{R}^{(3+n+k) \times(3+n+k),}
$$

where $\mathbf{M}_{p}$ and $\mathbf{M}_{p r}$ are defined in (1), the inertial terms of the elastically connected motors are summarized in

$$
\begin{aligned}
& \mathbf{D}_{K}=\operatorname{diag}\left\{\mathbf{D}_{K^{1}}, \mathbf{D}_{K^{2}}, \cdots, \mathbf{D}_{K^{m}}\right\} \in \mathbb{R}^{k \times k},
\end{aligned}
$$

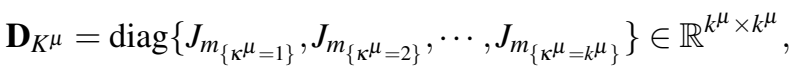

and $\mathbf{M}_{r E}$ is the link side inertia matrix, which is expressed as $\mathbf{M}_{r E}=\mathbf{M}_{r}-\mathbf{S}_{N} \mathbf{D}_{N}$, with $\mathbf{D}_{N}=\operatorname{diag}\left\{\mathbf{D}_{N^{1}}, \mathbf{D}_{N^{2}}, \cdots, \mathbf{D}_{N^{m}}\right\} \in$ $\mathbb{R}^{n \times n}$, where $\mathbf{D}_{N^{\mu}}=\operatorname{diag}\left\{J_{m_{1} \mu}, J_{m_{2} \mu}, \cdots, J_{m_{n} \mu}\right\} \in \mathbb{R}^{n^{\mu} \times n^{\mu}}$ is the matrix of the inertias of all motors of the $\mu$-th manipulator. The gravitational and Coriolis/centrifugal forces are

$$
\mathbf{g}_{E}=\left[\begin{array}{c}
\mathbf{g} \\
\mathbf{0}_{k \times 1}
\end{array}\right] \in \mathbb{R}^{(3+n+k)}, \quad \mathbf{c}_{E}=\left[\begin{array}{c}
\mathbf{c} \\
\mathbf{0}_{k \times 1}
\end{array}\right] \in \mathbb{R}^{(3+n+k)}
$$

where $\mathbf{g} \in \mathbb{R}^{(3+n)}$ is given in (2) and $\mathbf{c} \in \mathbb{R}^{(3+n)}$ is given in (3). The generalized forces are

$$
\begin{aligned}
& \mathbf{f}_{E}=\mathbf{G}_{E} \mathbf{u} \in \mathbb{R}^{(3+n+k)}, \mathbf{G}_{E}=\left[\begin{array}{c}
\mathbf{G}-\overline{\mathbf{S}}_{N} \\
\overline{\mathbf{S}}_{K}
\end{array}\right] \in \mathbb{R}^{(3+n+k) \times(n+2),} \\
& \overline{\mathbf{S}}_{N}=\left(\begin{array}{cc}
\mathbf{0}_{3 \times 2} & \mathbf{0}_{3 \times n} \\
\mathbf{0}_{n \times 2} & \mathbf{S}_{N} \in \mathbb{R}^{n \times n}
\end{array}\right), \overline{\mathbf{S}}_{K}=\left(\begin{array}{l}
\mathbf{0}_{k \times 2} \mathbf{S}_{K} \in \mathbb{R}^{k \times n}
\end{array}\right)
\end{aligned}
$$

where $\overline{\mathbf{S}}_{N} \in \mathbb{R}^{(n+3) \times(n+2)}, \quad \overline{\mathbf{S}}_{K} \in \mathbb{R}^{k \times(n+2)}$ and $\mathbf{G}$, u are from (5)-(6).

We denote with $f_{l_{v} \mu}\left(\theta_{0 v^{\mu}}, \theta_{m_{0 v^{\mu}}}\right)$ and $f_{m_{v^{\mu}}}\left(\theta_{0 v^{\mu}}, \theta_{m_{0 v^{\mu}}}\right)$ the link-side and the motor-side elastic forces for the $v^{\mu}$-th joint, respectively ${ }^{1}$. Those forces are identically zero if $v^{\mu} \notin K^{\mu}$. In the case $v^{\mu} \in K^{\mu}$ they are instead generic functions of $\theta_{0 v^{\mu}}, \theta_{m_{0 v^{\mu}}}$. In the linear spring case $f_{l_{v} \mu}\left(\theta_{0 v^{\mu}}, \theta_{m_{0 v^{\mu}}}\right)=k_{e_{v} \mu}\left(\theta_{m_{0 v^{\mu}}}-\theta_{0 v^{\mu}}\right)$ and $f_{m_{v} \mu}\left(\theta_{0 v^{\mu}}, \theta_{m_{0 v^{\mu}}}\right)=k_{e_{v^{\mu}}}\left(\theta_{0 v^{\mu}}-\theta_{m_{0 v^{\mu}}}\right)$ where $k_{e_{v^{\mu}}}>0$ is the stiffness of the elastic element. We then define

$$
\begin{aligned}
\mathbf{f}_{l^{\mu}} & =\left[\begin{array}{lll}
f_{l_{1} \mu}\left(\theta_{01^{\mu}}, \theta_{m_{01^{\mu}}}\right) & \cdots & f_{l_{n^{\mu}}}\left(\theta_{0 n^{\mu}}, \theta_{m_{0 n^{\mu}}}\right)
\end{array}\right]^{T} \in \mathbb{R}^{\mu^{\mu}}, \\
\mathbf{f}_{m^{\mu}} & =\left[\begin{array}{lll}
f_{m_{1} \mu}\left(\theta_{01^{\mu}}, \theta_{m_{01} \mu}\right) & \cdots & f_{m_{n^{\mu}}}\left(\theta_{0 n^{\mu}}, \theta_{m_{0 n^{\mu}}}\right)
\end{array}\right]^{T} \in \mathbb{R}^{n^{\mu}}, \\
\mathbf{f}_{L}\left(\mathbf{q}_{r}\right) & =\operatorname{diag}\left\{\mathbf{f}_{l^{1}}\left(\mathbf{q}_{r^{1}}\right), \mathbf{f}_{l^{2}}\left(\mathbf{q}_{r^{2}}\right), \cdots, \mathbf{f}_{l^{m}}\left(\mathbf{q}_{r^{m}}\right)\right\} \in \mathbb{R}^{n} \\
\mathbf{f}_{M}\left(\mathbf{q}_{r}\right) & =\operatorname{diag}\left\{\mathbf{f}_{m^{1}}\left(\mathbf{q}_{r^{1}}\right), \mathbf{f}_{m^{2}}\left(\mathbf{q}_{r^{2}}\right), \cdots, \mathbf{f}_{m^{m}}\left(\mathbf{q}_{r^{m}}\right)\right\} \in \mathbb{R}^{n}, \\
\mathbf{f}_{E l} & =\left[\begin{array}{lll}
\mathbf{0}_{1 \times 3} & \mathbf{f}_{L}^{T}\left(\mathbf{q}_{r}\right) \mathbf{S}_{N}^{T} & \mathbf{f}_{M}^{T}\left(\mathbf{q}_{r}\right) \mathbf{S}_{K}^{T}
\end{array}\right]^{T} \in \mathbb{R}^{(3+n+k)} .
\end{aligned}
$$

The system dynamics assumes then the following form

$$
\mathbf{M}_{E} \ddot{\mathbf{q}}+\mathbf{c}_{E}+\mathbf{g}_{E}=\mathbf{G}_{E} \mathbf{u}+\mathbf{f}_{E l} .
$$

The following result holds:

Proposition 2. $\mathbf{y}=\left[\begin{array}{ll}\mathbf{p}_{0}^{T} & \mathbf{q}_{r}^{T}\end{array}\right]^{T} \in \mathbb{R}^{(n+2)}$ is a flat output for the protocentric aerial manipulator with mixed rigid/elastic joints $(1 \leq k \leq n)$. The total relative degree is $4+4 \max _{\mu} \tilde{k}^{\mu}+\sum_{\mu=1}^{m}\left(2+2 \tilde{k}^{\mu}\right) n^{\mu}$, with $\tilde{k}^{\mu}=\max \left(1, k^{\mu}\right)$.

Proof. (Sketch) See Sec. I-B of [23] for the full proof and necessary remarks. Similar to the proof of Prop. 1, using the dynamics of the CoM positions of the overall system we can show that $\theta_{0}$ and $u_{t}$ are the sole functions of the flat outputs. Now, consider the $v^{\mu}$-th motor. If it is rigid, its torque is identical to (8). If it is elastic, then we first compute,

\footnotetext{
${ }^{1}$ In this paper we do not consider the frictions on the elastic connections.
}

$$
\begin{gathered}
\theta_{m_{0 v^{\mu}}}=\theta_{0 v^{\mu}}+\frac{1}{k_{e_{v^{\mu}}}}\left(\tau_{v^{\mu}+1}+\mathbf{m}_{0 v^{\mu}}^{T}\left(\theta_{0 v^{\mu}}\right) \ddot{\mathbf{p}}_{0}+\right. \\
+\sum_{l=1, l \neq v^{\mu}}^{n^{\mu}} m_{l v^{\mu}}\left(\theta_{0 l^{\mu}}, \theta_{0 v^{\mu}}\right) \ddot{\theta}_{0 l^{\mu}}+ \\
\left.+\left(\mathbb{J}_{v^{\mu}}-J_{m_{v^{\mu}}}\right) \ddot{\theta}_{0 v^{\mu}}+g_{r_{v^{\mu}}}\left(\theta_{0 v^{\mu}}\right)+c_{r_{v^{\mu}}}\left(\dot{\mathbf{q}}_{r^{\mu}}, \mathbf{q}_{r^{\mu}}\right)\right) .
\end{gathered}
$$

Notice the similarity with (8). We observe that (13) can also be employed for $v^{\mu}=n^{\mu}$, simply setting $\tau_{n^{\mu}+1}$ equal to zero. Then, $\tau_{v^{\mu}}$ can be easily computed from

$$
\tau_{v^{\mu}}=J_{v^{\mu}} \ddot{\theta}_{m_{0 v^{\mu}}}+k_{e_{v^{\mu}}} \theta_{m_{0 v^{\mu}}}-k_{e_{v^{\mu}}} \theta_{0 v^{\mu}} .
$$

From the third equation of the system dynamics we have

$$
u_{r}=\mathbb{J}_{0} \ddot{\theta}_{0}+\sum_{j=1}^{m} \tau_{1^{j}}-d_{G_{x}} u_{t},
$$

in which $\tau_{1}^{\mu}$ is taken from either (8) or (14), depending on the type of the actuation.

In order to briefly explain the relative degree formula, notice the different relative degree of the dependencies of $\tau_{v^{\mu}}$ given in (14) on the flat outputs for different values of $v^{\mu}$. Assume for instance that both the $\left(n^{\mu}-1\right)$-th and the $n^{\mu}$-th link are elastic. Then first, from (14) for $v^{\mu}=n^{\mu}$, we see that $\tau_{n^{\mu}}$ is a function of $\ddot{\theta}_{m_{0 n} \mu}$; while $\theta_{m_{0 n^{\mu}}}$ is a function of $\ddot{\mathbf{p}}_{0}$ and $\ddot{\mathbf{q}}_{r^{\mu}}$, making $\tau_{n^{\mu}}$ itself a function of $\dddot{\mathbf{p}}_{0}$ and $\dddot{\mathbf{q}}_{r^{\mu}}$. Second, from (14), $\tau_{n^{\mu}-1}$ is a function of $\ddot{\theta}_{m_{0\left(n^{\mu}-1\right)}}$. But in (13), from recursion, $\theta_{m_{0\left(n^{\mu}-1\right)}}$ is a function of $\tau_{n^{\mu}}$, making $\ddot{\theta}_{m_{0\left(n^{\mu}-1\right)}}$, and thus $\tau_{n^{\mu}-1}$, a function of $\ddot{\tau}_{n^{\mu}}$. Knowing from the first step above that $\tau_{n^{\mu}}$ is a function of $\dddot{\mathbf{p}}_{0}$ and $\dddot{\mathbf{q}}_{r^{\mu}}$, we find $\tau_{n^{\mu}-1}$ as a function of $\mathbf{p}_{0}^{(6)}$ and $\mathbf{q}_{r^{\mu}}^{(6)}$, which are the sixth time derivatives.

This can be generalized by recalling that $k^{\mu}$ is the number of elastic joints in link $\mu$, and defining $\tilde{k}^{\mu}=\max \left(1, k^{\mu}\right)$, then $r=4+4 \max _{\mu} \tilde{k}^{\mu}+\sum_{\mu=1}^{m}\left(2+2 \tilde{k}^{\mu}\right) n^{\mu}$, where it can be seen a quadratic dependence on the number of elastic joints. The term $\max _{\mu} \tilde{k}^{\mu}$ returns the value $\tilde{k}^{\mu}$ for the manipulator arm with the highest number of elastic joint. To fix the ideas, see also the examples in Sec. I-B of [23].

\section{Control for Case R}

In this section we present the exact linearizing controller for the system given in (7). We purposely limit our computation to the Case R, since the high relative degree involved in Case E may cause the controller to be unpractical.

Now, based on the findings of Proposition 1, we take $\mathbf{y}=\left[\mathbf{p}_{0}^{T} \mathbf{q}_{r}^{T}\right]^{T} \in \mathbb{R}^{(n+2)}$ as control variables, leaving out the PVTOL orientation $\theta_{0}$. We approach the control problem by studying the system with $\theta_{0}$ removed. We can decompose the inertia matrix $\mathbf{M}$ by defining the following quantities:

$$
\mathbf{M}=\left(\begin{array}{c:c|c}
\tilde{\mathbf{M}}_{p} & \mathbf{0} & \tilde{\mathbf{M}}_{p r}^{T} \\
\hdashline \mathbf{0} & J_{0} & \mathbf{0} \\
\hline \tilde{\mathbf{M}}_{p r} & \mathbf{0} & \mathbf{M}_{r}
\end{array}\right) \quad \tilde{\mathbf{M}}=\left(\begin{array}{c|c}
\tilde{\mathbf{M}}_{p} & \tilde{\mathbf{M}}_{p r}^{T} \\
\hline \tilde{\mathbf{M}}_{p r} & \mathbf{M}_{r}
\end{array}\right)
$$

where $\mathbf{0}$ is a zero vector or matrix of proper dimensions, $\tilde{\mathbf{M}}_{p}=\operatorname{diag}\left(m_{s} m_{s}\right)$, and $\tilde{\mathbf{M}}_{p r}$ is simply constituted by the first 
two columns of $\mathbf{M}_{p r}$. Similarly, for $\mathbf{G}$ :

$$
\mathbf{G}=\left(\begin{array}{c:c|c}
\mathbf{v} & \mathbf{0} & \mathbf{0} \\
\hdashline d_{G_{x}} & 1 & \mathbf{G}_{r p} \\
\hline \mathbf{0} & \mathbf{0} & \mathbf{G}_{r r}
\end{array}\right) \quad \tilde{\mathbf{G}}=\left(\begin{array}{c|c}
\mathbf{v} & \mathbf{0} \\
\hline \mathbf{0} & \mathbf{G}_{r r}
\end{array}\right)
$$

where $\mathbf{v}=\left[-\sin \theta_{0}-\cos \theta_{0}\right]^{T}$. This allows us to write:

$$
\tilde{\mathbf{M}}(\mathbf{y}) \ddot{\mathbf{y}}+\tilde{\mathbf{n}}(\mathbf{y}, \dot{\mathbf{y}})=\tilde{\mathbf{G}}\left(\theta_{0}\right) \tilde{\mathbf{u}},
$$

where $\tilde{\mathbf{n}}$ is $\mathbf{n}=\mathbf{c}+\mathbf{g}$ with the $3^{\text {rd }}$ element removed, and $\tilde{\mathbf{u}}=\left[u_{t} \boldsymbol{\tau}^{T}\right]^{T}$, where $\boldsymbol{\tau}=\left[\begin{array}{lll}\boldsymbol{\tau}^{1} & \cdots & \boldsymbol{\tau}^{m}\end{array}\right]^{T}$. Now we can differentiate (16), yielding (dependencies omitted):

$$
\tilde{\mathbf{M}} \ddot{\mathbf{y}}+\dot{\tilde{\mathbf{M}}} \ddot{\mathbf{y}}+\dot{\tilde{\mathbf{n}}}=\tilde{\mathbf{G}} \dot{\tilde{\mathbf{u}}}+\dot{\tilde{\mathbf{v}}} u_{t},
$$

where we have evidenced $\tilde{\mathbf{v}}=\left[\mathbf{v}^{T} \mathbf{0}\right]^{T}$. Differentiating further:

$$
\tilde{\mathbf{M}} \dddot{\mathbf{y}}+2 \dot{\tilde{\mathbf{M}}} \dddot{\mathbf{y}}+\ddot{\tilde{\mathbf{M}}} \ddot{\mathbf{y}}+\ddot{\tilde{\mathbf{n}}}=\tilde{\mathbf{G}} \ddot{\tilde{\mathbf{u}}}+2 \dot{\tilde{\mathbf{v}}} \dot{u}_{t}+\ddot{\tilde{\mathbf{v}}} u_{t},
$$

but:

$$
\ddot{\mathbf{v}}=\left[\begin{array}{c}
-\cos \left(\theta_{0}\right) \ddot{\theta}_{0} \\
\sin \left(\theta_{0}\right) \ddot{\theta}_{0}
\end{array}\right]+\left[\begin{array}{c}
\sin \left(\theta_{0}\right) \dot{\theta}_{0}^{2} \\
\cos \left(\theta_{0}\right) \dot{\theta}_{0}^{2}
\end{array}\right]=\mathbf{h} \ddot{\theta}_{0}-\mathbf{v} \dot{\theta}_{0}^{2},
$$

where $\mathbf{h}=\left[-\cos \left(\theta_{0}\right) \sin \left(\theta_{0}\right)\right]^{T}$. From the $3^{\text {rd }}$ row of (7)

$$
\ddot{\theta}_{0}=\frac{1}{J_{0}}\left(d_{G_{x}} u_{t}+u_{r}+\mathbf{G}_{r p} \boldsymbol{\tau}\right) \text {. }
$$

We substitute (20) in (19), and (19) in the last term of (18):

$$
\ddot{\mathbf{v}} u_{t}=\frac{u_{t}}{J_{0}} \mathbf{h} \cdot\left(d_{G_{x}} u_{t}+u_{r}+\mathbf{G}_{r p} \boldsymbol{\tau}\right)-\mathbf{v} u_{t} \dot{\theta}_{0}^{2}=\boldsymbol{\gamma}+\frac{u_{t}}{J_{0}} \mathbf{h} \cdot u_{r}
$$

where we have introduced the new symbol $\boldsymbol{\gamma}$ for compactness. This finally allows us to write:

$$
\tilde{\mathbf{M}} \dddot{\mathbf{y}}+2 \dot{\tilde{\mathbf{M}}} \dddot{\mathbf{y}}+\ddot{\tilde{\mathbf{M}}} \ddot{\mathbf{y}}+\ddot{\tilde{\mathbf{n}}}-2 \dot{\tilde{\mathbf{v}}} \dot{u}_{t}-\tilde{\boldsymbol{\gamma}}=\overline{\mathbf{G}} \overline{\mathbf{u}}
$$

where:

$$
\overline{\mathbf{G}}=\left(\begin{array}{c|c|c}
\mathbf{v} & u_{t} \mathbf{h} & \mathbf{0} \\
\hline \mathbf{0} & \mathbf{0} & \mathbf{G}_{r r}
\end{array}\right) \quad \tilde{\boldsymbol{\gamma}}=\left[\begin{array}{l}
\boldsymbol{\gamma} \\
\mathbf{0}
\end{array}\right] \quad \overline{\mathbf{u}}=\left[\begin{array}{c}
\ddot{u}_{t} \\
u_{r} \\
\ddot{\boldsymbol{\tau}}
\end{array}\right] .
$$

Matrix $\overline{\mathbf{G}} \in \mathbb{R}^{(2+n) \times(2+n)}$ is the decoupling matrix and it is clearly invertible, as long as $u_{t} \neq 0$, since $|\overline{\mathbf{G}}|=-\frac{u_{t}}{J_{0}}$.

The relative degree of the extended system is clearly $r=4(2+n)=8+4 n$. Notice the overall new states of the system can be described with $\overline{\mathbf{x}}=\left[\mathbf{q}^{T} \in \mathbb{R}^{(3+n)}, \dot{\mathbf{q}}^{T} \in \mathbb{R}^{(3+n)}, \tilde{\mathbf{u}}^{T} \in \mathbb{R}^{(1+n)}, \dot{\tilde{\mathbf{u}}}^{T} \in \mathbb{R}^{(1+n)}\right]^{T} \in \mathbb{R}^{\bar{n}}$, meaning that the total number of states is $\bar{n}=2(3+n)+2(1+n)=8+4 n=r$. Thus, no internal dynamics is left, consistently with the notion that the system is flat. The virtual control input can be computed as

$$
\overline{\mathbf{u}}=\overline{\mathbf{G}}^{-1}\left(\tilde{\mathbf{M}} \dddot{\mathbf{y}}_{r}+2 \dot{\tilde{\mathbf{M}}} \dddot{\mathbf{y}}+\ddot{\tilde{\mathbf{M}}} \ddot{\mathbf{y}}+\ddot{\tilde{\mathbf{n}}}-2 \dot{\tilde{\mathbf{v}}} \dot{u}_{t}-\tilde{\boldsymbol{\gamma}}\right),
$$

where, for a desired $\mathbf{y}$ trajectory denoted as $\mathbf{y}_{d}$

$$
\begin{aligned}
\dddot{\mathbf{y}}_{r}= & \dddot{\mathbf{y}}_{d}+\mathbf{K}_{3}\left(\dddot{\mathbf{y}}_{d}-\dddot{\mathbf{y}}\right)+\mathbf{K}_{2}\left(\ddot{\mathbf{y}}_{d}-\ddot{\mathbf{y}}\right) \\
& +\mathbf{K}_{1}\left(\dot{\mathbf{y}}_{d}-\dot{\mathbf{y}}\right)+\mathbf{K}_{0}\left(\mathbf{y}_{d}-\mathbf{y}\right)+\mathbf{K}_{-1} \int_{0}^{t}\left(\mathbf{y}_{d}-\mathbf{y}\right) \mathrm{d} t .
\end{aligned}
$$

The $\mathbf{K}_{i}$ 's are diagonal positive definite matrices, assigned according to the usual linear pole-placement strategies. Specifically, if $K_{i, j}$ is the $j$-th diagonal element of $\mathbf{K}_{i}$, then each polynomial

$$
p_{j}(x)=x^{5}+K_{3, j} x^{4}+K_{2, j} x^{3}+K_{1, j} x^{2}+K_{0, j} x+K_{-1, j}
$$
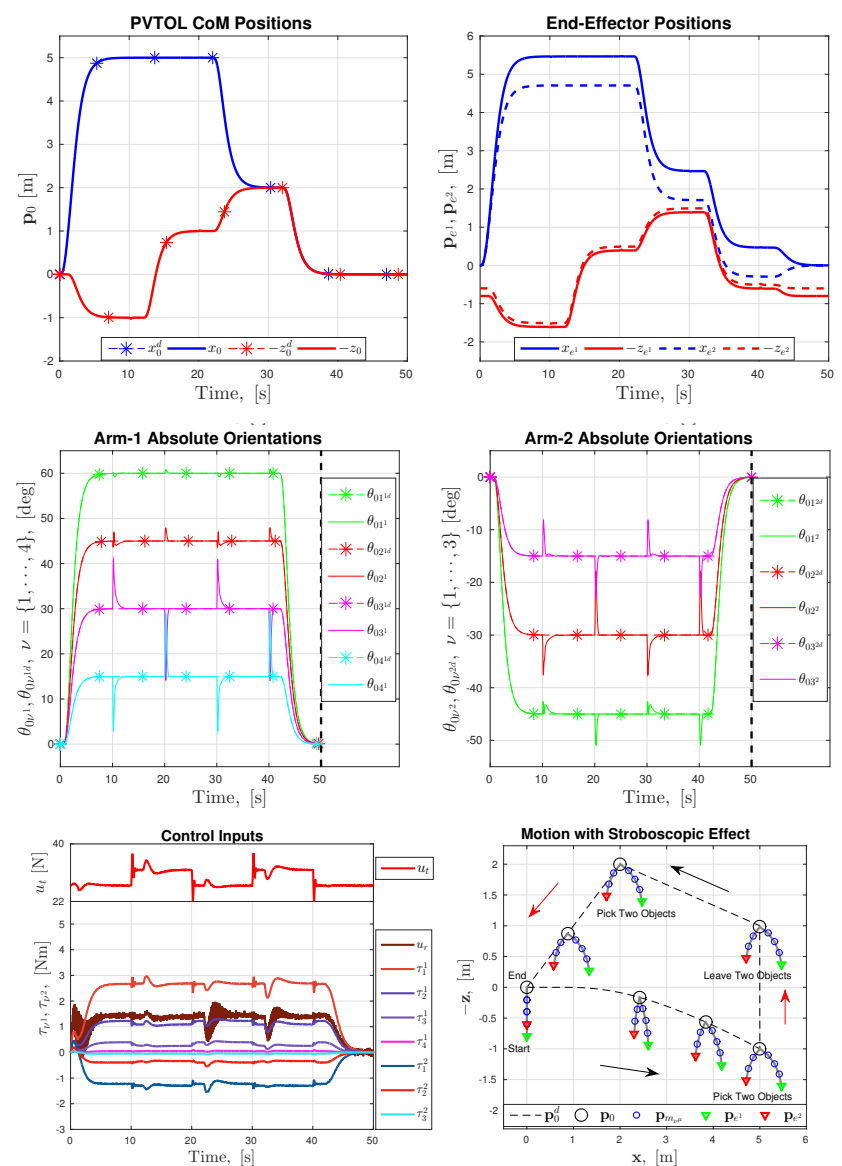

Fig. 3: Simulation results of a pick and place task. The effect of the unknown grasped masses on the end-effector positions is negligible. In the bottom right, the arrows show the direction of the motion. Red arrows correspond to the mass-carrying phases. In the last plot, the thrust input is given on the top, separately. More simulation results are given in [23] and in the video attachment.

must be Hurwitz, i.e. all its roots must have negative real parts; the introduction of an integral error term provides some ability to reject disturbances, such as carried loads and parameter uncertainty (see Sec. VI for its implementation). The inverse of $\overline{\mathbf{G}}$ is easily obtained:

$$
\overline{\mathbf{G}}^{-1}=\left(\begin{array}{cc|c}
-\sin \theta_{0} & -\cos \theta_{0} & \mathbf{0} \\
-\frac{J_{0}}{u_{t}} \cos \theta_{0} & \frac{J_{0}}{u_{t}} \sin \theta_{0} & \\
\hline \mathbf{0} & \mathbf{G}_{r r}^{-1}
\end{array}\right)
$$

$$
\mathbf{G}_{r r}^{-1}=\left(\begin{array}{ccc}
\mathbf{G}_{r r^{1}}^{-1} & & \mathbf{0} \\
& \ddots & \\
\mathbf{0} & & \mathbf{G}_{r r^{m}}^{-1}
\end{array}\right) \quad \mathbf{G}_{r r^{i}}^{-1}=\left(\begin{array}{ccc}
1 & \cdots & 1 \\
& \ddots & \vdots \\
& & 1
\end{array}\right) \in \mathbb{R}^{n^{i} \times n^{i}}
$$

It should be noticed, the algorithm makes apparent use of higher-order derivatives of the flat outputs, $\ddot{\mathbf{y}}$ and $\dddot{\mathbf{y}}$, which are difficult or impossible to estimate directly. However, these can be computed from $\tilde{\mathbf{u}}$ and $\dot{\tilde{\mathbf{u}}}$, obtained from integration of appropriate components of the virtual input $\overline{\mathbf{u}}$ :

$$
\begin{aligned}
\ddot{\mathbf{y}} & =\tilde{\mathbf{M}}^{-1}(\tilde{\mathbf{G}} \tilde{\mathbf{u}}-\tilde{\mathbf{n}}) \\
\dddot{\mathbf{y}} & =\tilde{\mathbf{M}}^{-1}\left(\tilde{\mathbf{G}} \dot{\tilde{\mathbf{u}}}+\dot{\tilde{\mathbf{v}}} u_{t}-\dot{\tilde{\mathbf{M}}} \ddot{\mathbf{y}}-\dot{\tilde{\mathbf{n}}}\right) .
\end{aligned}
$$




\section{NUMERICAL VALIDATION}

In this section we present simulations results for testing the proposed controller in a realistic situation in which measurement noises and sampling errors are taken into account We consider a PAM with $m=2, n^{1}=4$ and $n^{2}=3$. Hence the flat output is $\mathbf{y}=\left[\begin{array}{lllllll}\mathbf{p}_{0} & \theta_{01^{1}} & \cdots & \theta_{04^{1}} & \theta_{01^{2}} & \cdots & \theta_{03^{2}}\end{array}\right]^{T} \in \mathbb{R}^{9}$. System and simulation parameters are given in Table I of [23]. A pick and place task is chosen for the robot. This is divided in 5 phases: $i$ ) the robot follows a desired trajectory, ii) the two arms grasp two individual point mass objects with unknown mass for the controller (each mass is $0.25 \mathrm{~kg}$ ), iii) the objects are carried to another location, where they are unloaded, $i v$ ) phase (i) and (ii) are repeated while following a different trajectory, $v$ ) phase (iii) is repeated while following a different trajectory, and then arms return to the initial configuration. We encourage the reader to watch the attached video for a better understanding.

Notice that the tracking performance is almost perfect, despite the uncertainties. At the time of grasping there are small errors on the tracking of the flat outputs, which are due to the unknown masses. However these errors goes to zero again thanks to the integral terms defined in the controller (see Sec. V), and their effects on both PVTOL CoM and end-effector positions are negligible.

\section{CONCLUSION}

In this paper we have introduced a particularly relevant class of aerial manipulators that we named protocentric. We have shown that protocentric aerial manipulators are differentially flat systems regardless of the number of arms and the presence of rigid- or elastic-joints in the arms. We have then proposed a controller for the case of rigid joints only and we have validated the controller with simulations.

In this study we observed that if the aerial vehicle is underactuated, the number of the compliant actuators increases the relative degree quadratically. This requires much smoother trajectories to be tracked, which would eventually lead to very slow robot motions. Hence choosing a fully actuated aerial vehicle might be beneficial if it is equipped with an arm that has multiple compliant joints. We will investigate this further in our future studies.

In the future we also plan also to extend our theory to the $3 \mathrm{D}$ case, to use of sensor-based calibration methods as, e.g., in [24] to retrieve the system parameters on the fly, and to investigate the use of decentralized multi-robot schemes [25][28] for the control of multiple aerial manipulators.

\section{REFERENCES}

[1] ARCAS, "EU Proj. ICT-287617," www.arcas-project.eu, 2011-2015.

[2] AeRoArms, "EU Collab. Project ICT-644271," www.aeroarms-project. eu, 2015-2019.

[3] D. J. Lee and C. Ha, "Mechanics and control of quadrotors for tool operation," in 2012 ASME Dynamic Systems and Control Conference, Fort Lauderdale, FL, Oct. 2012.

[4] H.-N. Nguyen, S. Park, and D. J. Lee, "Aerial tool operation system using quadrotors as rotating thrust generators," in 2015 IEEE/RSJ Int. Conf. on Intelligent Robots and Systems, Hamburg, Germany, Oct. 2015, pp. 1285-1291.

[5] S. Kim, S. Choi, and H. J. Kim, "Aerial manipulation using a quadrotor with a two dof robotic arm," in 2013 IEEE/RSJ Int. Conf. on Intelligent Robots and Systems, Tokyo, Japan, November 2013, pp. 4990-4995.
[6] H. Yang and D. J. Lee, "Dynamics and control of quadrotor with robotic manipulator," in 2014 IEEE Int. Conf. on Robotics and Automation, Hong Kong, China, May. 2014.

[7] K. Kondak, F. Hubert, M. Schwarzbach, M. Laiacker, D. Sommer, M. Bejar, and A. Ollero, "Aerial manipulation robot composed of an autonomous helicopter and a 7 degrees of freedom industrial manipulator," in 2014 IEEE Int. Conf. on Robotics and Automation, Hong Kong, China, May 2014, pp. 2108-2112.

[8] H. Baizid, G. Giglio, F. Pierri, M. A. Trujillo, G. Antonelli, F. Caccavale, A. Viguria, S. Chiaverini, and A. Ollero, "Experiments on behavioral coordinated control of an unmanned aerial vehicle manipulator system," in 2015 IEEE Int. Conf. on Robotics and Automation, Seattle, USA, May. 2015.

[9] C. Korpela, M. Orsag, and P. Oh, "Towards valve turning using a dualarm aerial manipulator," in 2014 IEEE/RSJ Int. Conf. on Intelligent Robots and Systems, Chicago, USA, Sep. 2014.

[10] B. Yüksel, S. Mahboubi, C. Secchi, H. H. Bülthoff, and A. Franchi, "Design, identification and experimental testing of a light-weight flexible-joint arm for aerial physical interaction," in 2015 IEEE Int. Conf. on Robotics and Automation, Seattle, WA, 2015, pp. 870-876.

[11] B. Yüksel, N. Staub, and A. Franchi, "Aerial robots with rigid/elasticjoint arms: Single-joint controllability study and preliminary experiments," in 2016 IEEE/RSJ Int. Conf. on Intelligent Robots and Systems, Daejeon, South Korea, Oct. 2016.

[12] B. Siciliano and O. Khatib, Handbook of Robotics. Springer, 2008.

[13] R. M. Murray, M. Rathinam, and W. Sluis, "Differential flatness of mechanical control systems: A catalog of prototype systems," in ASME Int. Mechanical Eng. Congress and Exposition, CA, Nov. 1995.

[14] M. Fliess, J. Levine, P. Martin, and P. Rouchon, "A Lie-Bäcklund approach to equivalence and flatness of nonlinear systems," IEEE Trans. on Automatic Control, vol. 44, no. 5, pp. 922-937, 1999.

[15] B. Siciliano, L. Sciavicco, L. Villani, and G. Oriolo, Robotics: Modelling, Planning and Control. Springer, 2009.

[16] A. De Luca and W. Book, "Robots with flexible elements," in Springer Handbook of Robotics, B. Siciliano and O. Khatib, Eds. Springer, 2008, pp. 287-319.

[17] A. De Luca, "Decoupling and feedback linearization of robots with mixed rigid/elastic joints," in 1996 IEEE Int. Conf. on Robotics and Automation, Minneapolis, USA, Apr. 1996, pp. 816-821.

[18] S. Haddadin and K. Krieger, "On impact decoupling properties of elastic robots and time optimal velocity maximization on joint level," in 2012 IEEE/RSJ Int. Conf. on Intelligent Robots and Systems, Vilamoura, Portugal, October 2012, pp. 5089-5096.

[19] V. Mistler, A. Benallegue, and N. K. M'Sirdi, "Exact linearization and noninteracting control of a 4 rotors helicopter via dynamic feedback," in 10th IEEE Int. Symp. on Robots and Human Interactive Communications, Bordeaux, Paris, France, Sep. 2001, pp. 586-593.

[20] J. Thomas, J. Polin, K. Sreenath, and V. Kumar, "Avian-Inspired Grasping for Quadrotor Micro UAVs," in 2013 ASME Int. Design Engineering Technical Conf. and Computers and Information in Engineering Conf., Portland, OR, Aug. 2013.

[21] S. Lupashin, A. Schöllig, M. Sherback, and R. D'Andrea, "A simple learning strategy for high-speed quadrocopter multi-flips," in 2010 IEEE Int. Conf. on Robotics and Automation, Anchorage, AK, May 2010, pp. 1642-1648.

[22] S. Lupashin and R. D'Andrea, "Stabilization of a flying vehicle on a taut tether using inertial sensing," in 2013 IEEE/RSJ Int. Conf. on Intelligent Robots and Systems, Japan, Nov 2013, pp. 2432-2438.

[23] B. Yüksel, G. Buondonno, and A. Franchi, "Explicit proofs and computations," LAAS-CNRS, Tech. Rep. hal-01349878, July 2016. [Online]. Available: https://hal.archives-ouvertes.fr/hal-01349878

[24] A. Censi, A. Franchi, L. Marchionni, and G. Oriolo, "Simultaneous maximum-likelihood calibration of odometry and sensor parameters," IEEE Trans. on Robotics, vol. 29, no. 2, pp. 475-492, 2013.

[25] A. Franchi, P. Stegagno, M. Di Rocco, and G. Oriolo, "Distributed target localization and encirclement with a multi-robot system," in 7th IFAC Symp. on Intell. Auton. Vehicles, Lecce, Italy, Sep. 2010.

[26] J. W. Durham, A. Franchi, and F. Bullo, "Distributed pursuit-evasion with limited-visibility sensor via frontier-based exploration," in 2010 IEEE ICRA, Anchorage, AK, May 2010, pp. 3562-3568.

[27] A. Franchi, G. Oriolo, and P. Stegagno, "Probabilistic mutual localization in multi-agent systems from anonymous position measures," in 49th IEEE CDC, Atlanta, GA, Dec. 2010, pp. 6534-6540.

[28] _ - "Mutual localization in multi-robot systems using anonymous relative measurements," The International Journal of Robotics Research, vol. 32, no. 11, pp. 1302-1322, 2013. 